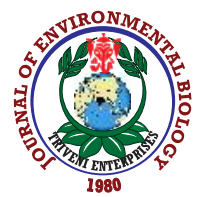

\title{
Molecular characterization of phosphate solubilizing fungi Aspergillus niger and its correlation to sustainable agriculture
}

\author{
Y.S. Mazrou ${ }^{1,2 *}$, A.H. Makhlouf', E.R. Elbealy', M.A. Salem ${ }^{5}$, M.A. Farid ${ }^{6}$, M.F. Awad ${ }^{7,8}$, M.M. Hassan $^{7,9}$ and M. Ismail ${ }^{9}$ \\ ${ }^{1}$ Department of Business Administration Department, Community College, King Khaled University, Abha, 62529, Saudi Arabia \\ ${ }^{2}$ Department of Agric. Economics, Faculty of Agriculture, Tanta University, Tanta, 3111, Egypt \\ ${ }^{3}$ Department of Botany, Faculty of Agriculture, Menoufia University, Menoufia, 32514, Egypt \\ ${ }^{4}$ Department of Biology, Faculty of Science, King Khalid University, Abha, 61413, SaudiArabia \\ ${ }^{5}$ Department of Chemistry, Faculty of Science and Art, King Khalid University, Asir, 61421, Saudi Arabia \\ ${ }^{6}$ Department of Biology, Faculty of Science, Taif University, Taif, 21944, Saudi Arabia \\ ${ }^{7}$ Department of Genetics, Faculty of Agriculture, Kafrelsheikh University, Kafr El-Sheikh,33516, Egypt \\ ${ }^{8}$ Department of Botany and Microbiology, Faculty of Science, Al-azhar University, Assuit, 71511, Egypt \\ ${ }^{9}$ Department of Genetics, Faculty of Agriculture, Menoufia University, Menoufia, 32514, Egypt \\ *Corresponding Author Email : mazrou_y@yahoo.com
}

\begin{tabular}{lll}
\hline Paper received: 14.10 .2019 & Revised received: 15.01 .2020 & Accepted: 28.02 .2020 \\
\hline
\end{tabular}

\section{Abstract}

Aim: The present study aimed to investigate the phosphate solubilization potential of agriculturally important fungi, i.e., Aspergillus sp. isolated from the rhizosphere of healthy plants in Abha city, Saudi Arabia.

Methodology: Sixteen Aspergillus sp. isolated and tested for phosphate solubilization potential were identified by $5.8 S$-ITS region sequencing and characterized by 11 ISSRPCR markers. Finally, the highest phosphate solubilization potential isolates were used in field experiments on cucumber and tomato plants.

Results: All Aspergillus niger isolates showed $96-100 \%$ similarity to A. niger strains available at GenBank database, Isolate ASAB-5 was most efficient at solubilizing phosphate on Pikovskaya's medium, with a solubilization index of 2.67 , and $235.22 \mathrm{mgl}^{-1}$ of solubilized phosphate. ISSR-PCR markers revealed is total 142 bands in all isolates, with about $32.3 \%$ showing monomorphism and $67.6 \%$ polymorphism. Based on genetic similarity and intraspecies variability, the Aspergillus isolates were grouped into two different clusters with about $67.9 \%$ genetic similarity. The results of field experiments showed no significant difference between seeds treated with culture filtrate or conidial suspension of ASAB-5; however, both differed remarkably from untreated seeds.

Interpretation: The current study confirms the existence of several useful phosphate solubilizing fungi in plants, which may serve as potential biological fertilizers. They are safer than chemical fertilizers and increase the bioavailability of soil phosphates for plants.

Key words: Aspergillus niger, ISSR markers, Phosphate solubilization, 5.8S-ITS sequencing

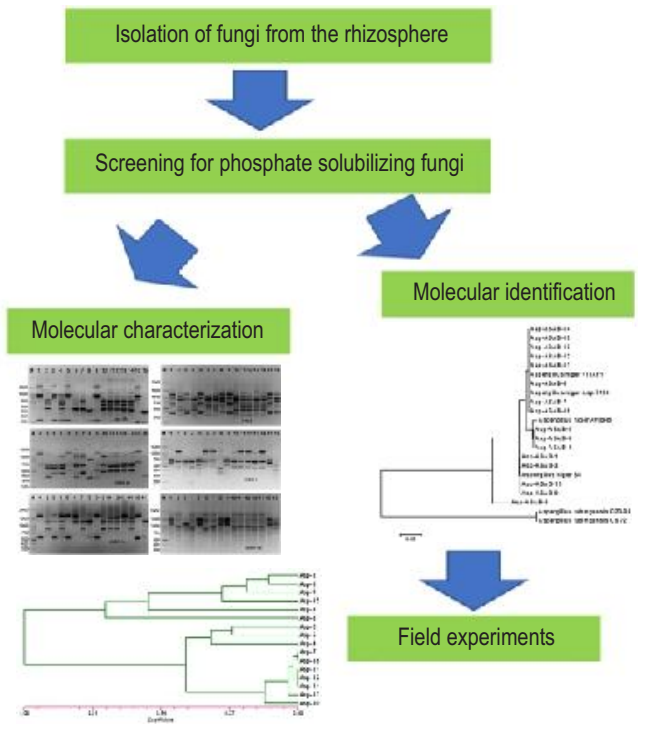

How to cite : Mazrou, Y.S., A.H. Makhlouf, E.R. Elbealy, M.A. Salem, M.A. Farid, M.F. Awad, M.M. Hassan and M. Ismail: Molecular characterization of phosphate solubilizing fungi Aspergillus niger and its correlation to sustainable agriculture. J. Environ. Biol., 41, 592-599 (2020). 


\section{Introduction}

Phosphate is one of the most important macronutrients for plant growth and development. However, in soil, it is relatively found in low concentration, ranging from 500 to $10,000 \mathrm{~kg} \mathrm{P}$ in the upper parts of the soil. Phosphorus compounds commonly found in soils are mostly unavailable for plant uptake, as they are highly insoluble complex (Son et al., 2006; Baig et al., 2010; Chauhan et al., 2017). When soluble sources of phosphorus, such as fertilizers and manures, are added to soils, they may become fixed (changed to unavailable forms) and in time form highly insoluble compounds (Khan et al., 2010; Gupta and Pandey, 2019). Some farmers address this problem by using phosphate fertilizers, which become incorporated in the soil. Unfortunately, this practice is costly, environmentally troublesome, and may lead to insolubility of phosphorus (Dawwam et al., 2013). Moreover, only $5 \%$ of the added phosphorus is actually available to plants (Khan et al., 2010; Pande et al., 2017). As a result, chemical fertilizers are not suitable for regular use in agricultural operations. Phosphate solubilizing microbes, which convert phosphate into a form suitable for plant uptake, represent a safe, as well as cheap and environmentally friendly alternative to chemical-based phosphate fertilizers (Sharma et al., 2013). Some fungi, such as Aspergillus sp. ( $A$. awamori, $A$. niger, $A$. tereus, $A$. flavus, $A$. nidulans, $A$. foetidus, and $A$. wentii) and Penicillium sp. ( $P$. digitatum, $P$. lilacinium, $P$. balaji, and $P$. funicolosum) are known phosphate solubilizing microbes (Chuang et al., 2007; Pandey et al., 2008; Jain et al., 2012).

Identification of most common and important species of the genus Aspergillus remains problematic due to variability in the phenotypic characters (Nayak et al., 2019). However, a validated and careful approach of phenotypic classification (taxonomy) together with phylogenetic treatment of DNA sequence data is a prerequisite for a reliable and rapid identification (Khan et al., 2007; Godet and Munaut, 2010; Manjunath et al., 2017). Recently, advancements in molecular techniques have created new possibilities for the selection and genetic improvement of live fungal stocks. Internal transcribed spacer (ITS) region on rDNA, in particular, is the most useful method for inter- and intraspecific sequence-based identification of Aspergillus genus and other fungi (Toju et al., 2012; Riaz et al., 2017; Hassan et al., 2019). In view of the above, the present study aimed to investigate the phosphate solubilization potential of Aspergillus sp. isolated from the rhizosphere of healthy plants in Abha city, Saudi Arabia.

\section{Materials and Methods}

Soil samples: Twelve different tomato rhizospheric soil samples were collected from farms situated in different areas of Abha city, and surrounding locations. The collected soil samples were brought to the laboratory, dried, and stored for further analyses.

Isolation of fungi from the rhizosphere: All soil samples were subjected to serial dilution in potato dextrose agar (PDA) medium for isolation of fungi. The fungi were identified based on the morphological, conidial and culturing characteristics, and then identified at molecular level using 5.8S-ITS region sequencing.

Screening for phosphate solubilizing fungi: To identify phosphate solubilizing fungi, the isolates were cultured separately on Pikovskaya's agar plates and incubated at $28 \pm 2{ }^{\circ} \mathrm{C}$ for 7 days. The ability of fungi to produce a halo/clear zone due to solubilization was selected as an indicator of potential phosphate solubilizers. Phosphate solubilization index (SI) was calculated by the formula (Pikovskaya, 1948) given below:

\section{$\mathrm{SI}=($ Colony diameter + Halo diameter $) /$ Colony diameter}

DNA extraction: Fungal mycelia of selected phosphate solubilizers were inoculated into Czapex Dox broth and incubated for 5 days. Genomic DNA of each selected fungus was extracted using the Norgen Plant/Fungi DNA isolation Kit (Sigma, Canada) following manufacturer's instructions.

Sequence analysis of 5.8S-ITS region: ITS1 and ITS4 primers were designed according to White et al. (1990). PCR products were sequenced by Macrogen International Co. Seoul, Korea. The produced sequences were aligned and nucleotide BLAST tool (http://www.ncbi.nlm.nih.gov/BLAST/) was selected to determine the homology between the obtained fungal sequences and related sequences in the GenBank database. MEGA software version 7.0 was used to draw a phylogenetic tree of the obtained sequences and related $\mathrm{NCBI}$ sequences.

Inter simple sequence repeats (ISSR)-PCR analysis: For repetitive sequence analysis, PCR of selected fungal isolates was performed according to Lakhani et al. (2016). Eleven ISSR primers (ISSR-Asp, ISSR-G4, ISSR-G5, ISSR-2, ISSR-8, ISSR9, ISSR-10, ISSR-12, ISSR-16, ISSR-18, and ISSR-28) were used to amplify the genomic DNA. A simple matching coefficient was estimated by means of Jaccard's coefficient and was used to construct a similarity matrix by NTSYS-pc, ver. 2.2 .

Molecular characterization of selected fungi: Total DNA extracted from the Aspergillus sp. isolates were used for PCR amplification of fragments in the calmodulin gene. Specifically, NIG1/NIG2 and TUB1/TUB2 primer pairs were used to discriminate between $A$. niger and $A$. tubingensis. The extracted DNA was used for detecting phosphate solubilization genes, glucose dehydrogenase $(g d h)$, and its cofactor pyrroloquinoline-quinone synthase (pqq). Finally, total DNA of these isolates were used to assess the presence of Ota and Aopks genes and production of ochratoxin A (Ferracin et al., 2012). (Primers are listed in Table 1).

Field experiments: Seeds of cucumber (Sweet Success variety) and tomato (Super Sweet 100 variety) were sterilized for 2 min by sodium hypochlorite and rinsed several times in sterile distilled water. They were then soaked in a conidial suspension or culture filtrate of Aspergillus sp. ASAB- 5 at $1 \times 10^{8}$ colony-forming units per ml. (Murali et al., 2016). Treated and untreated seeds (three 
replicates of ten seeds) were planted and grown in pots for 30 days to evaluate shoot and root fresh weight, dry weight and length.

Statistical analyses: Data from three replicates were analyzed by Analysis of Variance using SPSS Inc. 16.0 software (IBM, USA). Significant differences were calculated according to Duncan's Multiple Range Test (Steel and Torrie, 1980). Means within a column followed by same letter were not significantly different at $p=0.05$ level.

\section{Results and Discussion}

In total 106 fungal isolates were collected from the rhizosphere of different plants in the Abha region of southern Saudi Arabia. Thirty-nine of them were classified as Aspergillus $\mathrm{sp}$. Their colonies grew rapidly on PDA, and initially their white floccose mycelium spread rapidly, turning quickly into blackcolored colonies that produced black spores (Godet and Munaut, 2010; Nayak et al., 2019). Four isolates of Fusarium sp., whose mycelia were of dirt white color, justly dense, and with yellow pigmentation at the periphery, were also found. Moreover, the mycelia grew in thick concentric rings. Some isolates displayed bright pink pigmentation at the center (Gaddeyya et al., 2012).

Fourteen of them were recognized as Penicillium sp., because their colonies were typically fast growing, in shades of green, occasionally white, and consisted a dense felt of conidiophores (Pandey et al., 2008). Sixteen isolates were identified as Trichoderma sp. when grown on PDA; they formed 1-2 concentric rings with green conidia, which were highly dense in the center and then spread to the margins (Hassan et al., 2014). Eighteen isolates were identified as Pythium sp.; their mycelia grew densely and presented a cotton appearance, especially on rich medium such as PDA (Gaddeyya et al., 2012). Finally, 15 isolates were identified as Alternaria sp.; their conidia were of muri-form shape and light brown color (Hassan et al., 2014). The isolates were serially numbered and stored prior to the phosphate solubilization experiment. All isolates were tested for phosphate solubilization using Pikovskaya's agar medium method. Fungi that rapidly formed clear areas and were of the largest diameter indicated a superior phosphate solubilizing capacity. Among the tested fungi, 16 isolates of Aspergillus sp. exhibited the highest phosphate solubilization value and were selected for estimating phosphate SI. The SI of different isolates ranged from 1.79 to 2.67 after 7 days of incubation at $28-30^{\circ} \mathrm{C}$. As depicted in Table 2 , ASAB- 5 was the most efficient phosphate solubilizing Aspergillus sp. on Pikovskaya's medium with SI value of 2.67, followed by $A S A B-13$ with SI value of 1.96 and $A S A B-11$ with SI value of 1.96; whereas the lowest SI (of 1.79) was detected in ASAB-9 (Table 2). Moreover, ASAB-5 yielded the highest amount of solubilized phosphate $\left(235 \mathrm{mg} \mathrm{l}^{-1}\right)$, followed by ASAB-2 (180.52 $\left.\mathrm{mg} \mathrm{l}^{-1}\right)$; whereas ASAB-9 was the least efficient one $\left(171.36 \mathrm{mg} \mathrm{l}^{-1}\right)$. Current results are in agreement with the earlier findings (Richa et al., 2007; Khan et al., 2010; Onyia et al., 2015), where high incidence of phosphate solubilizing fungi were reported belonged to the genus Aspergillus and to a lesser extent to genus Penicillium spp. in the rhizosphere of several crop plants.

Two universal oligonucleotides (ITS1 and ITS4) were used to amplify the 5.8S-ITS region in Aspergillus sp. isolates. The primers successfully amplified a 600-bp fragment from the 5.8S-ITS region in all phosphate solubilizing Aspergillus sp. isolates. Comparing incomplete 5.8S-ITS region sequence with these in GenBank database revealed that all 16 isolates corresponded to A. niger. Phylogenetic analysis based on 5.8SITS sequencing classified the isolates into two main taxonomic lineages (Fig. 1). The first main lineage contained three sublineages. The first one comprised Aspergillus isolates ASAB-10, ASAB-11, ASAB-12, ASAB-13, and ASAB-14 with $100 \%$ similarity to $A$. niger VITAF1; as well as ASAB-7, ASAB-8 and ASAB-16 with $100 \%$ similarity to $A$. niger Asp-7136. The second sub-lineage contained Aspergillus isolates ASAB-2, ASAB-4 and ASAB- 6 with $98 \%$ similarity to $A$. niger AHBR5. Finally, the third sub-lineage contained Aspergillus isolates ASAB-1, ASAB-3, ASAB-5, ASAB9 and ASAB-15 with $96-100 \%$ similarity to $A$. niger S5. The second main lineage contained two $A$. tubingensis isolates, G2005 and $\mathrm{Cs} 72$, which displayed $92 \%$ similarity to other $A$. niger isolates. ITS rDNA profiling offers a cost-effective method for

Table 1: PCR primers used to detect target genes (NIG, TUB, gdh, pqq, Ota, and Aopks) in phosphate solubilizing Aspergillus sp. isolates

\begin{tabular}{|c|c|c|c|}
\hline Primers name & Primer sequence $\left(5^{\prime}->3^{\prime}\right)$ & Size (bp) & References \\
\hline NIG1 & TCCGTAGGTGAACCTGCGG & 245 & Susca et al. (2007) \\
\hline NIG2 & GCTGCGTTCTTCATCGATGC & & \\
\hline TUB1 & GCATCGATGAAGAACGCAGC & 505 & Susca et al. (2007) \\
\hline TUB2 & TCCTCCGCTTATTGATATGC & & \\
\hline gdh-f & ATGTATCCGGATTTAAAAGG & 798 & Chauhan et al. (2017) \\
\hline gdh-r & TTAACCGCGGCCTGCCTGG & & \\
\hline pqq- $f$ & GGCTGCTGGCCGAACTGACTT & 1000 & Chauhan et al. (2017) \\
\hline pqq-r & GGCCGCAAGAAGCATTATTAG & & \\
\hline OTAF & CAATGCCGTCCAACCGTATG & 776 & Ferracin et al. (2012) \\
\hline OTAF & CCTTCGCCTCGCCCGTAG & & \\
\hline Aopks-F & CAGACCATCGACACTGCATGC & 530 & Ferracin et al. (2012) \\
\hline Aopks-R & CTGGCGTTCCAGTACCATGAG & & \\
\hline
\end{tabular}


Table 2: Phosphate solubilization index (PSI) and amount of phosphate solubilized by the isolates of Aspergillus sp.

\begin{tabular}{lll}
\hline Isolates name & PSI & Solubilized $\mathbf{P}\left(\mathrm{mg} \mathrm{l}^{-1}\right)$ \\
\hline ASAB-1 & $1.92^{\mathrm{c}}$ & $175.16^{\mathrm{d}}$ \\
ASAB-2 & $1.95^{\mathrm{b}}$ & $180.52^{\mathrm{b}}$ \\
ASAB-3 & $1.91^{\mathrm{c}}$ & $178.91^{\mathrm{b}}$ \\
ASAB-4 & $1.94^{\mathrm{b}}$ & $179.47^{\mathrm{b}}$ \\
ASAB-5 & $2.67^{\mathrm{a}}$ & $235.22^{\mathrm{a}}$ \\
ASAB-6 & $1.84^{\mathrm{e}}$ & $172.42^{\mathrm{e}}$ \\
ASAB-7 & $1.83^{\mathrm{e}}$ & $172.61^{\mathrm{e}}$ \\
ASAB-8 & $1.92^{\mathrm{c}}$ & $175.15^{\mathrm{d}}$ \\
ASAB-9 & $1.79^{\mathrm{f}}$ & $171.36^{\mathrm{f}}$ \\
ASAB-10 & $1.87^{\mathrm{d}}$ & $174.71^{\mathrm{d}}$ \\
ASAB-11 & $1.96^{\mathrm{b}}$ & $178.11^{\mathrm{b}}$ \\
ASAB-12 & $1.94^{\mathrm{b}}$ & $177.57^{\mathrm{c}}$ \\
ASAB-13 & $1.95^{\mathrm{b}}$ & $178.74^{\mathrm{b}}$ \\
ASAB-14 & $1.89^{\mathrm{d}}$ & $176.32^{\mathrm{d}}$ \\
ASAB-15 & $1.85^{\mathrm{e}}$ & $172.94^{\mathrm{e}}$ \\
ASAB-16 & $1.95^{\mathrm{b}}$ & $177.58^{\mathrm{c}}$ \\
\hline
\end{tabular}

Values within the same vertical area with same letter are not significantly differentat $5 \%$ probability level by Duncan's Multiple Range Test

identifying and characterizing complex microbial communities at species level, following morphological and cultural examination (Rastogi and Sani, 2011; Rao et al., 2015). To confirm the identity of our isolates and discriminate between $A$. niger and $A$. tubingensis, a specific PCR assay targeting DNA sequence of calmodulin gene (Susca et al., 2007) was used. The primer set N1G1/N1G2 amplified only DNA from $A$. niger strains, whereas the primer set TUB1/TUB2 amplified only DNA belonging to $A$. tubingensis (Susca et al., 2007). The specific primer pair NIG1/NIG2 amplified a 265-bp fragment in all isolates, whereas primers TUB1/TUB2 failed to amplify any region, strongly suggesting that all strains belonged to $A$. niger. PCR amplification of phosphate solubilization genes, such as gdh and pqq (Son et al., 2006), yielded no product for the latter but produced a 798-bp gdh fragment with isolates ASAB-2, ASAB-4, ASAB-5, ASAB-6, ASAB-7, ASAB-10, and ASAB-13. Finally, we used primers against Ota and $A$ opks to ensure that ASAB-5, which were selected for field experiments, did not produce any ochratoxin and was safe to use. The Ota gene was amplified in most isolates exceptASAB2, ASAB-3, ASAB-4, ASAB-5, and ASAB-16. The Aopks gene was also found in some isolates, such as ASAB-6, ASAB-7, ASAB-11, ASAB-12 and ASAB-14. These results indicated that $A S A B-5$, the isolate with highest phosphate $\mathrm{SI}$, did not contain the ochratoxin gene and was suitable for field experiments.

The PCR-based ISSR markers method using arbitrary oligonucleotide primers is commonly employed for genetic analysis and as a screening genotyping method. ISSR-PCR markers have been validated as genetic markers in bacteria and fungi fingerprinting (Hassan et al., 2014). ISSR markers can produce numerous fingerprint patterns with infinite number of primers. Here, 11 different ISSR primers were tested in duplicate on DNA samples of Aspergillus sp. isolates and all the amplified products were reproducible. Products' size ranged from about $190 \mathrm{bp}$ to $2000 \mathrm{bp}$. Specifically, GTG5 primer produced in total 18 bands in all Aspergillus isolates, ranging from $250 \mathrm{bp}$ to $1950 \mathrm{bp}$. Six shared bands were detected in all Aspergillus isolates, exhibiting about 37.5\% monomorphism; whereas another 12 fragments showed $66.7 \%$ polymorphism (Table 3; Fig. 2). The ISSR-28 primer produced in total 12 fragments with about $50 \%$ polymorphism and 50\% monomorphism among sixteen Aspergillus isolates. The molecular size amplified products ranged from $350 \mathrm{bp}$ to $1900 \mathrm{bp}$.

According to genetic similarity and intraspecies differentiation, the Aspergillus isolates were grouped into two different clusters with about $67.9 \%$ genetic similarity. Based on these data, sixteen isolates were separated into two main clusters. Cluster 1 was divided into two sub-clusters; the first subcluster included Aspergillus isolates no. 1, 3, 5, 9 and 15, which displayed $56 \%$ similarity whereas the second sub-cluster included isolate no. 8. Cluster 2 was also divided into two sub-

Table 3: Polymorphic bands produced by each of the 11 ISSR-PCR primers and percentage of polymorphism in sixteen isolates of Aspergillus sp.

\begin{tabular}{llllll}
\hline Primers & Total bands & $\begin{array}{l}\text { No. of monomorphic } \\
\text { bands }\end{array}$ & $\begin{array}{l}\text { No. polymorphic } \\
\text { bands }\end{array}$ & $\begin{array}{l}\text { \% monomorphic } \\
\text { bands }\end{array}$ & \% Polymorphic \\
\hline ISSR-Asp & 16 & 6 & 10 & 37.5 & 62.5 \\
ISSR-G4 & 14 & 5 & 9 & 35.7 & 64.3 \\
ISSR-GTG5 & 18 & 6 & 12 & 33.3 & 66.7 \\
ISSR-2 & 9 & 3 & 6 & 33.3 & 66.7 \\
ISSR-8 & 15 & 5 & 10 & 33.3 & 66.7 \\
ISSR-9 & 16 & 4 & 12 & 25.0 & 75.0 \\
ISSR-10 & 11 & 3 & 8 & 27.3 & 81.7 \\
ISSR-12 & 11 & 2 & 9 & 18.2 & 66.7 \\
ISSR-16 & 6 & 2 & 4 & 33.3 & 71.4 \\
ISSR-18 & 14 & 4 & 10 & 28.6 & 50.0 \\
ISSR-28 & 12 & 6 & 6 & 50.0 & 67.6 \\
\hline Total & 142 & 46 & 96 & 32.3 & \\
\hline
\end{tabular}


Table 4: Effect of seed treatment with Aspergillus sp. ASAB-5 on cucumber plant

\begin{tabular}{lllllll}
\hline Treatment & \multicolumn{2}{c}{ Length $(\mathrm{cm})$} & \multicolumn{2}{c}{ Fresh weight $(\mathrm{gm})$} & \multicolumn{2}{c}{ Dry weight $(\mathrm{gm})$} \\
\cline { 2 - 7 } & Shoot & Root & Shoot & Root & Shoot & Root \\
\hline Culture filtrate & $12.0^{\mathrm{a}}$ & $15.2^{\mathrm{a}}$ & $2.92^{\mathrm{a}}$ & $1.33^{\mathrm{b}}$ & $0.36^{\mathrm{a}}$ & $0.09^{\mathrm{a}}$ \\
Conidial suspension & $11.9^{\mathrm{a}}$ & $15.0^{\mathrm{a}}$ & $2.85^{\mathrm{b}}$ & $1.46^{\mathrm{a}}$ & $0.36^{\mathrm{a}}$ & $0.10^{\mathrm{a}}$ \\
Control & $8.30^{\mathrm{b}}$ & $10.7^{\mathrm{b}}$ & $1.58^{\mathrm{c}}$ & $0.39^{\mathrm{c}}$ & $0.19^{\mathrm{b}}$ & $0.05^{\mathrm{b}}$ \\
\hline
\end{tabular}

Values within same vertical area with same letter are not significantly different at $5 \%$ probability level by Duncan's Multiple Range Test

Table 5: Effect of seed treatment with Aspergillus sp. ASAB-5 on tomato plant

\begin{tabular}{lllllll}
\hline Treatment & \multicolumn{2}{l}{ Shoot and Root length $(\mathrm{cm})$} & \multicolumn{2}{l}{ Fresh weight $(\mathrm{gm})$} & \multicolumn{2}{l}{ Dry weight (gm) } \\
\cline { 2 - 7 } & Shoot length & Root length & Shoot weight & Root weight & Shoot weight & Root weight \\
\hline Culture filtrate & $12.7^{\mathrm{b}}$ & $8.2^{\mathrm{b}}$ & $1.52^{\mathrm{a}}$ & $0.60^{\mathrm{a}}$ & $0.13^{\mathrm{a}}$ & $0.07^{\mathrm{a}}$ \\
Conidial suspension & $13.0^{\mathrm{a}}$ & $9.0^{\mathrm{a}}$ & $1.36^{\mathrm{b}}$ & $0.41^{\mathrm{b}}$ & $0.17^{\mathrm{a}}$ & $0.06^{\mathrm{a}}$ \\
Control & $8.50^{\mathrm{c}}$ & $6.2^{\mathrm{c}}$ & $0.57^{\mathrm{c}}$ & $0.14^{\mathrm{c}}$ & $0.04^{\mathrm{b}}$ & $0.02^{\mathrm{b}}$ \\
\hline
\end{tabular}

Values within the same vertical area with the same letter are not significantly different at $5 \%$ probability level by Duncan's Multiple Range Test

clusters; the first comprised isolates no. 2, 4, and 6 with $30 \%$ similarity, and the second isolates no. $7,10,11,12,13$, and 14 with $14 \%$ similarity (Fig. 3). All treated cucumber and tomato plants were harvested after 30 days, for studying growth parameters. Cucumber plants treated with culture filtrate of Aspergillus sp. ASAB-5 exhibited increase in all growth parameters as compared to control plants. The percent increase was $44.5 \%, 85 \%$ and $89.5 \%$ for shoot length, shoot fresh weight and shoot dry weight, respectively (Table 4). Similarity, cucumber plants treated with conidial suspension of Aspergillus sp. ASAB- 5 showed increase in shoot parameters. The percentage increase was $43.4 \%, 80 \%$, and $89.5 \%$ for shoot length, shoot fresh weight and shoot dry weight, respectively. Similar effet was also observed on the roots when treated with culture filtrate and conidial suspension of Aspergillus sp. ASAB-5. Using culture filtrate, the percentage increase in roots parameters was $42 \%, 2.4 \%$ and $80 \%$ for root

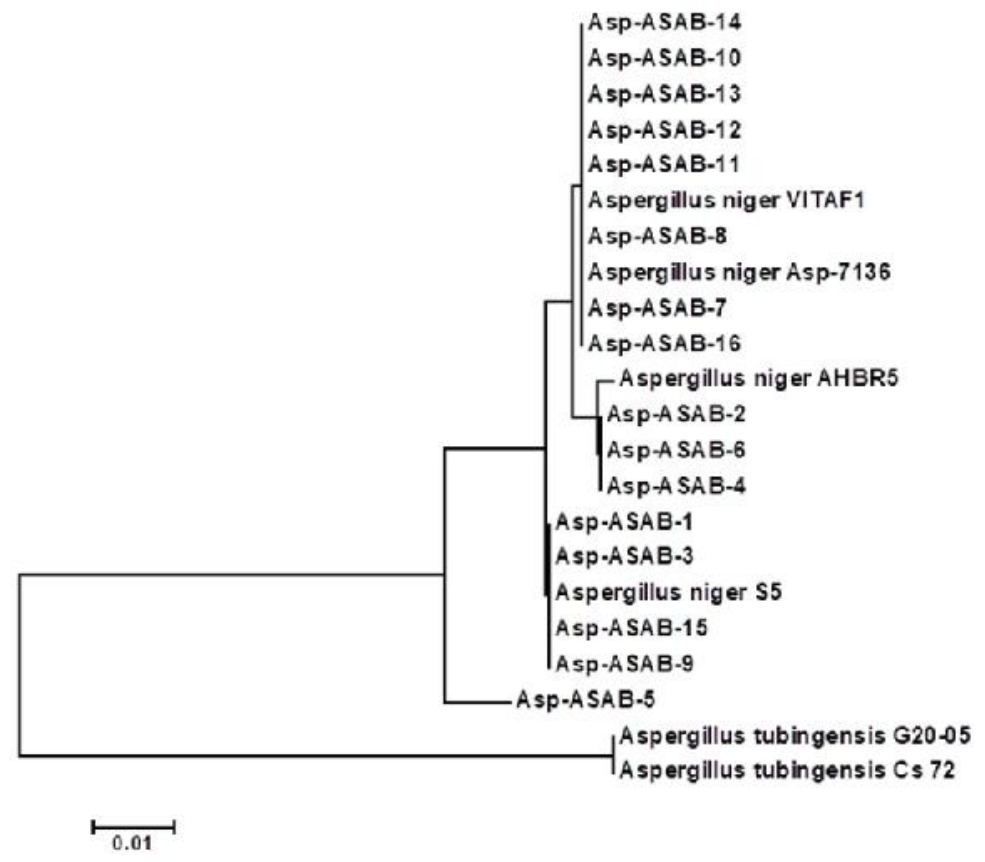

Fig. 1: Phylogenetic tree and diversity of 5.8S-ITS region in sixteen isolates of Aspergillus sp. compared with reference Aspergillus strains. Phylogenetic tree was generated using parsimony neighbor-joining and maximum likelihood analysis. 

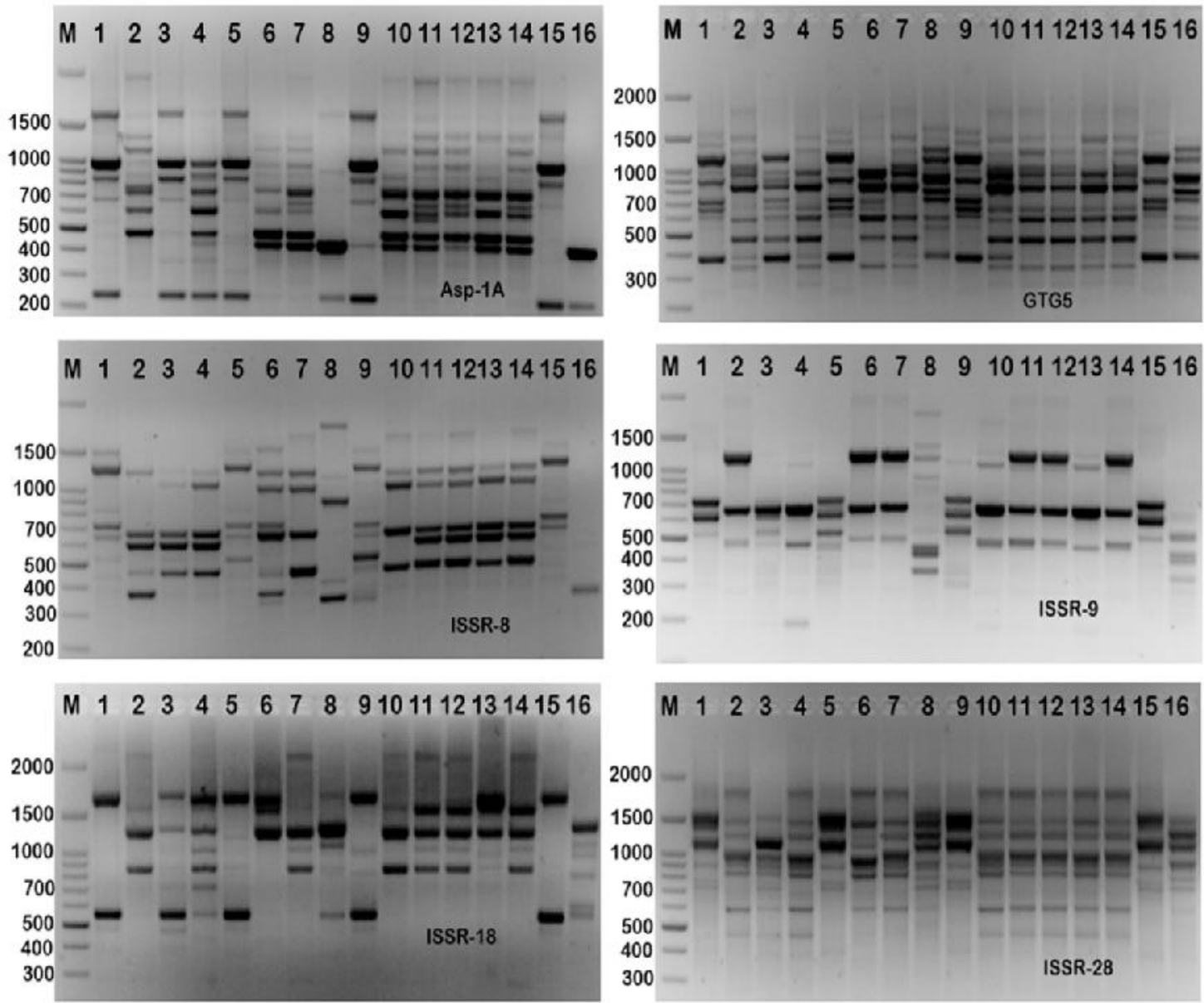

Fig. 2: ISSR-PCR profile of sixteen isolates of Aspergillus sp. generated with the respective ISSR primers: ISSR-Asp, GTG5, ISSR-8, ISSR-9, ISSR-18, and ISSR-28. The first lane in each panel corresponds to 100-bp molecular weight markers.

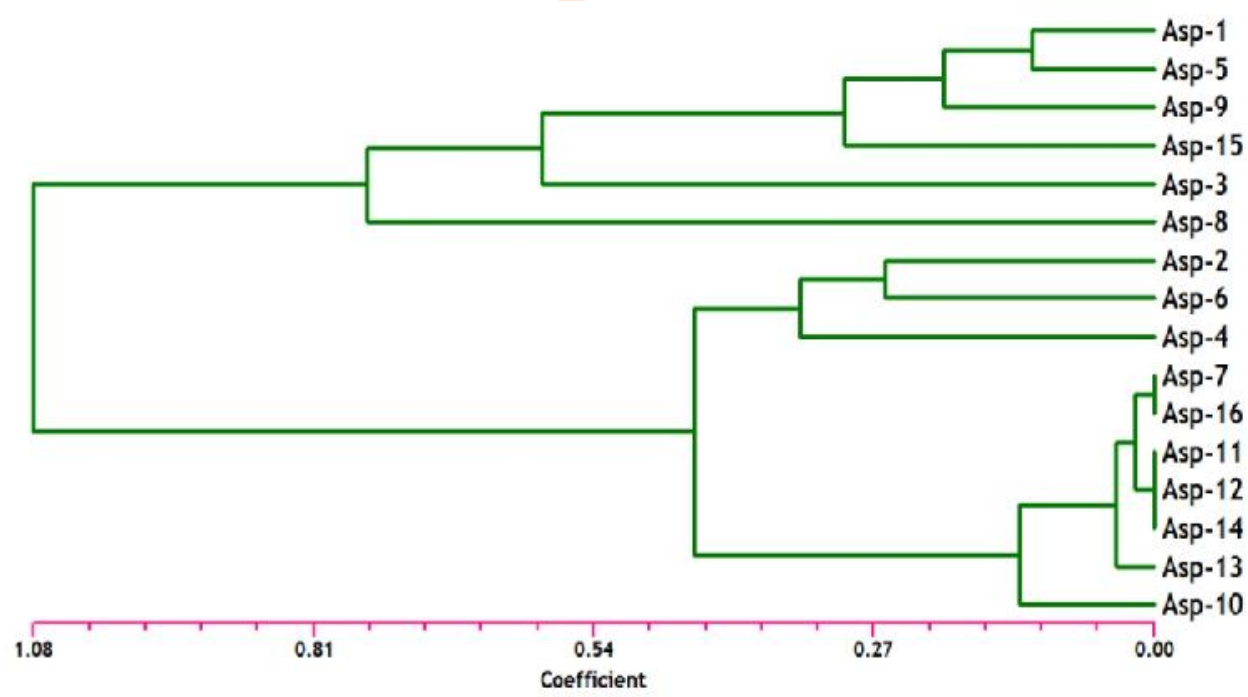

Fig. 3: Dendrogram analysis of sixteen isolates of Aspergillus sp. based on 11 ISSR-PCR primers. 
length, root fresh weight and root dry weight. Meanwhile, using Aspergillus sp. ASAB- 5 conidial suspension, the percent increase in root parameters was $40 \%, 2.74 \%$ and $100 \%$ for root length, root fresh weight and, root dry weight, respectively. Similar effect was also observed on tomato plants treated with culture filtrate and conidial suspension of Aspergillus sp. ASAB-5. Tomato plants treated with culture filtrate showed $49 \%, 1.7 \%$ and $2.25 \%$ increase in shoot length, shoot fresh weight and shoot dry weight. Tomato plants treated with conidial suspension also showed $53 \%, 1.4 \%$ and $2 \%$ increment in shoot length, shoot fresh weight and shoot dry weight, respectively. Similar effect was also observed on tomato roots when treated with culture filtrate and conidial suspension. Using culture filtrate, tomato roots showed $32 \%, 3.3 \%$ and $2.5 \%$ increment in root length, root fresh weight and root dry weight, respectively. Also using conidial suspension the increase in roots parameters was $45 \%, 2 \%$ and $2 \%$. These results corrborates with the study of Fuad et al. (2019), Phosphate-solubilizing bacteria and fungi are present in the soil. Si addition increased phosphate solubilization of fungi by $50 \%$. Statistical analysis revealed a significant difference $(p<0.05)$ between treated and untreated seeds, but no remarkable variance $(p<0.05)$ was observed between the seeds exposed to culture filtrate or conidial suspension of Aspergillus sp. ASAB-5. Thus, these experiments confirmed a significant enhancement in shoot fresh weight, dry weight and length in cucumber and tomato plants treated with phosphate solubilizing Aspergillus sp. ASAB-5.

A significant increase in tomato shoot fresh weight, dry weight and length is in line with previous reports Sudisha et al. (2013), who reported improved germination and seedling vigor in tomato seeds soaked with phosphate solubilizing fungi, such as Trichoderma, Penicillium, or Aspergillus. Similarly, A. niger and Penicillium italicum have been described to solubilize tricalcium phosphate and improve growth of soybean plants exposed to tricalcium phosphate-containing soil (Iman and Azouni, 2008; Eramma et al. , 2020)). Tomato seeds treated with Trichoderma harzianum (Fahmi et al., 2012) as well as pearl millet treated with Penicillium chrysogenum and Penicillium oxalicum (Murali et al., 2016) isolated from soil rhizosphere have shown improved seed germination and seedling vigor over control plants.

The present study compared the phosphate solubilizing potential of Aspergillus niger strains isolated from different farm locations in Abha city, Saudi Arabia. Phosphate solubilizing efficiency was highest in isolate ASAB-5 and was confirmed by the presence of phosphate solubilization genes. Importantly, this isolate significantly improved growth parameters of cucumber and tomato plants under field trials, suggesting the potential of such fungi for boosting crop yields.

\section{Acknowledgment}

This research was supported by the Research Support Program Deanship of Scientific Research King Khalid University, SaudiArabia (No. G.R.P-184-39).

\section{References}

Chauhan, A., G. Shiwani, P.B. Praveen, W. Abhishek, M. Rishi, M. Preeti and K.S. Chand: Tricalcium phosphate solubilization and nitrogen fixation by newly isolated Aneurinibacillus aneurinilyticus CKMV1 from rhizosphere of Valeriana jatamansi and its growth promotional effect. Braz. J. Microbiol., 48, 294-304 (2017).

Dawwam, G.E., A. El-Beltagy, H.M. Emara and M.M. Hassan: Beneficial effect of plant growth promoting bacteria isolated from the roots of potato plant. Annals Agricul. Sci., 58, 195-201 (2013).

Eramma, M., S. Rao, Y.M. Ramesh and N.M. Naik: Isolation and screening of phosphate solubilizing bacteria from paddy Rhizosphere Soil. Int. J. Curr. Microbiol. App. Sci., 9, 477-485 (2020).

Fuad, A., S.A. A.I.Yahya, S. AlNadhari, H. Alasmari, F. Alhoshani and M. Wainwright: Phosphate solubilizing bacteria and fungi in desert soils: Species, limitations and mechanisms. Arch. Agron. Soil Sci., 65, 1446-1459 (2019).

Fahmi, A.I., A.D. Al-Talhi and M.M. Hassan: Protoplast fusion enhances antagonistic activity in Trichoderma sp. Nat. Sci., 10, 100-106 (2012).

Ferracin, L.M., C.B. Fier, M.L. Carneiro-Vieira, C.B. Monteiro-Vitoriello, A. Mello-Varani, M.M. Rossi, M. Müller- Santos, M. HiromiTaniwaki, B. Thie- lamenaka and M.H. Pelegrinelli- Fungaro: Strain-specific polyketide synthase genes of Aspergillus niger. Int. J. Food Microbiol., 155, 137-145 (2012).

Gaddeyya, G., P.S. Niharika, P. Bharathi and P.K.R. Kumar: Isolation and identification of soil mycoflora in different crop fields at Salur Mandal. Adv. Appl. Sci. Res., 3, 2020-2026 (2012).

Godet, F. and F. Munaut: Molecular strategy for identification in Aspergillus section flavi. FEMS Microbiol. Lett., 304,157-168 (2010).

Gupta, S. and S. Pandey: ACC Deaminase producing bacteria with multifarious plant growth promoting traits alleviates salinity stress in French bean (Phaseolus vulgaris) Plants Front. Microbiol., 10,1506 (2019).

Hassan, M.M., A. Gaber and E.I. El-Hallous: Molecular and morphological characterization of Trichoderma harzianum from differentEgyptian soils. Wulfenia J., 21, 80-96 (2014).

Hassan, M.M., M.A. Farid and A. Gaber: Rapid identification of Trichoderma koningiopsis and Trichoderma longibrachiatum using sequence characterized amplified region markers. Egyp. J. Biol. Pest. Cont., 29,13 (2019).

Iman, M. and E.I. Azouni: Effect of phosphate solubilizing fungi on growth and nutrient uptake of soyabean plants. J. Appl. Sci. Res., 4, 592-598 (2008).

Jain, R., J. Saxena and V. Sharma: Solubilization of inorganic phosphates by Aspergillus awamori $\mathrm{S} 19$ isolated from agricultural soil of semi-arid region. Annals Microbiol., 62, 725-735(2012).

Khan, M.R., M.A. Anwer and F.A. Mohiddin: Molecular diversity in Aspergillus isolates collected from pigeonpea field in Aligarh region. Environ. Biol. Conserv., 12, 59-64. (2007).

Khan, M.S., A. Zaidi, M. Ahemad, M. Oves and P.A. Wani: Plant growth promotion by phosphate solubilizing fungi-current perspective. Arch. Agron. Soil Sci., 56, 73-98 (2010).

Lakhani, H.N., N.V. Dinesh, M.M. Hassan and R.A. Eissa: Fingerprinting and molecular comparison among two parental strains of Trichoderma spp. and their corresponding fusants produced by protoplast fusion. Biotechnol. Biotechnol. Equip., 30, 1065-1074 (2016).

Ltaief, B., B. Sifi, M. Zaman-Allah, J. Drevon and L. Mokhtar: Effect of salinity on root-nodule conductance to the oxygen diffusion in the Cicer arietinum, Mesorhizobium ciceri symbiosis. J. Plant Physiol., 164, 1028-1036 (2007). 
Manjunath, M., A. Singh, A.N. Tripathi, R. Prasanna, A.B. Rai and B. Singh: Bioprospecting the fungicides compatible Trichoderma asperellum isolate effective against multiple plant pathogens in vitro. J. Environ. Biol., 38, 553-560 (2017).

Murali, M., K.N. Amruthesh, J. Sudisha, S.R. Niranjana and H.S. Shetty: Screening for plant growth promoting fungi and their ability for growth promotion and induction of resistance in pearl millet against downy mildew disease. J. Phytol., 4, 30-36 (2012).

Murali, M., M.C. Thriveni, S. Manjula, S.R. Mythrashree and K.N. Amruthesh: Isolation of phosphate solubilizing fungi from rhizosphere soil and its effect on seed growth parameters of different crop plants. J. App. Biol. Biotech., 4, 022-026 (2016).

Nayak, S., U. Dhua and S. Samanta: Morphological and toxigenic study of Aspergillus flavus from indoor air. J. Environ. Biol., 40, 648-654 (2019).

Onyia, C.E., C.U. Anyawu and M.N. Ikegbunam: Ability of fungi, isolated from nsukka peppers and garden-egg plant rhizospheres, to solubilize phosphate and tolerate cadmium. Adv. Microbiol., 5, 500-506 (2015).

Pande, A., P. Prashant, M. Simmi, S. Mritunjay and K. Suresh: Phenotypic and genotypic characterization of phosphate solubilizing bacteria and their efficiency on the growth of maize. J. Genet. Eng. Biotechnol., 15, 379-391 (2017).

Pandey, A., N. Das, B. Kumar, K. Rinu and P. Trivedi: Phosphate solubilization by Penicillium spp. isolated from soil samples of Indian Himalayan region. World J. Microbiol. Biotechnol., 24, 97-102 (2008).

Pikovskaya, R.I.: Mobilization of phosphorus in soil in connection with vital activity of some microbial species. Mikrobiologiya, 17, 362-370 (1948).

Rao, H.Y., S. Baker, D. Rakshith and S. Satish: Molecular profiling and antimicrobial potential of endophytic Gliomastix polychroma CLB32 inhabiting Combretum latifolium Blume. Mycology, 6, 176-181 (2015).
Rastogi, G. and R.K. Sani: Molecular techniques to assess microbial community structure, function, and dynamics in the environment. In: Microbes and Microbial Technology: Agricultural and Environmental Applications (Eds.: I. Ahmad, F. Ahmad and J. Pichtel). Springer, New York, pp. 29-57 (2011).

Riaz, A., Z. Khalid, H.A. Rasib and R. Shahid: Molecular identification of Aspergillus flavus using inter transcribed spacers (ITS). Biotechnol. J. Int., 20, 1-8 (2017).

Richa, G., B. Khosla and M. Reddy: Improvement of maize plant growth by phosphate solubilizing fungi in rock phosphate amended soils. World J. Agricul. Sci., 3, 481-484 (2007).

Sharma, S.B., R.Z. Sayyed, M.H. Trivedi and T.A. Gobi: Phosphate solubilizing microbes: Sustainable approach for managing phosphorus deficiency in agricultural soils. Springer Plus, 2, 587 (2013).

Son, H.J., G.T. Park, M.S. Cha and M.S. Heo: Solubilization of insoluble inorganic phosphates by a novel salt and $\mathrm{pH}$ tolerant Pantoea agglomerans R-42 isolated from soybean rhizosphere. Bioresour. Technol., 97, 204-210 (2006).

Steel, R.B. and J.H. Torrie: Principles and Procedures of Statistics. $2^{\text {nd }}$ Edn., Mc-Graw-Hill. Inc., New York, USA, p. 633 (1980).

Sudisha, J., M. Abdelrahman, P.T. Lam-Son and S. Ito: Characterization of rhizosphere fungi that mediate resistance in tomato against bacterial wilt disease. J. Exper. Bot., 64, 3829-3842 (2013).

Susca, A., G. Stea, G. Mulè and G. Perrone: PCR identification of Aspergillus niger and Aspergillus tubingensis based on calmodulin gene. FoodAddit. Contam., 24, 1154-1160 (2007).

Toju, H., A.S. Tanabe, S. Yamamoto and H. Sato: High-coverage ITS primers for the DNA based identification of ascomycetes and basidiomycetes in environmental samples. PLoS ONE, 7, e40863 (2012).

White, T.J., T. Bruns, S. Lee and J. Taylor: Amplification and direct sequencing of fungal ribosomal RNA genes for phylogenetics. PCR protocols. Guid. Meth. Appl., 18, 315-322 (1990). 